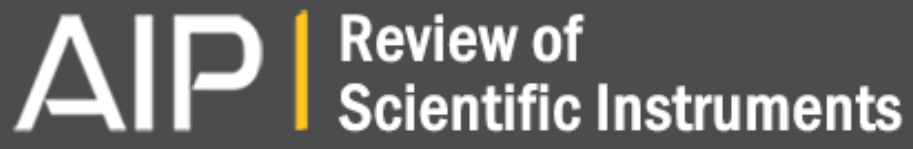

\section{High resolution autofocus for spatial temporal biomedical research}

Sihong Li, Xiaodong Cui, and Wei Huang

Citation: Review of Scientific Instruments 84, 114302 (2013); doi: 10.1063/1.4829616

View online: http://dx.doi.org/10.1063/1.4829616

View Table of Contents: http://scitation.aip.org/content/aip/journal/rsi/84/11?ver=pdfcov

Published by the AIP Publishing

\section{Articles you may be interested in}

A microdevice for the creation of patent, three-dimensional endothelial cell-based microcirculatory networks Biomicrofluidics 5, 034115 (2011); 10.1063/1.3609264

A benchtop, ultrafast infrared spectroscopic imaging system for biomedical applications

Rev. Sci. Instrum. 80, 123702 (2009); 10.1063/1.3262499

Fully automated, quantitative, noninvasive assessment of collagen fiber content and organization in thick collagen gels

J. Appl. Phys. 105, 102042 (2009); 10.1063/1.3116626

Bioengineering and Imaging Research Opportunities Workshop V: A white paper on imaging and characterizing structure and function in native and engineered tissues

Med. Phys. 35, 3428 (2008); 10.1118/1.2948317

Integrated structural and functional optical imaging combining spectral-domain optical coherence and multiphoton microscopy

Appl. Phys. Lett. 88, 053901 (2006); 10.1063/1.2171477

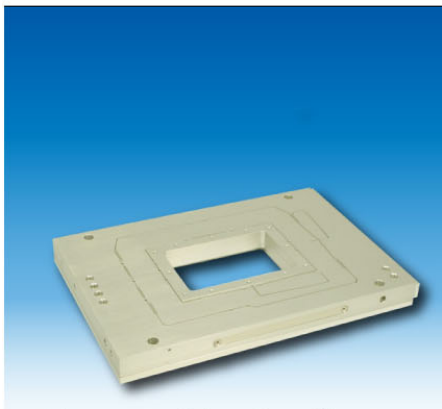

Nanopositioning Systems

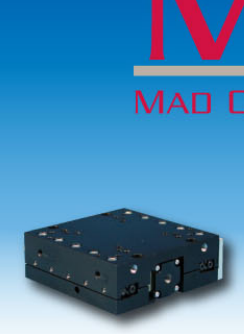

Micropositioning

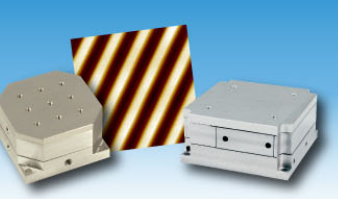

AFM \& SPM

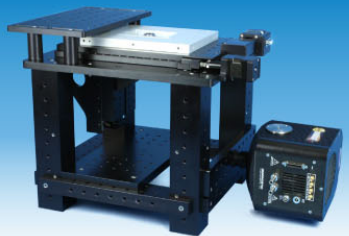

Single molecule imaging 


\title{
High resolution autofocus for spatial temporal biomedical research
}

\author{
Sihong Li, ${ }^{1}$ Xiaodong Cui, ${ }^{1}$ and Wei Huang ${ }^{1,2, a)}$ \\ ${ }^{1}$ Department of Physics, The University of Hong Kong, Pokfulam, Hong Kong \\ ${ }^{2}$ Department of Biochemistry, The University of Hong Kong, Pokfulam, Hong Kong and South University of \\ Science and Technology of China, Shenzhen, China
}

(Received 3 July 2013; accepted 23 October 2013; published online 14 November 2013)

\begin{abstract}
Maintaining focus has been a critical but challenging issue in optical microscopy, particularly for microscopic imaging systems currently used in biomedical research. During live cell imaging, environmental temperature fluctuations and other factors contribute to the unavoidable focus drift. For single molecular imaging and super resolution, focus drift can be significant even over short durations. The current commercial and experimental solutions are either optically complicated, expensive, or with limited axial resolution. Here, we present a simple autofocus solution based on low cost solid state laser and imaging sensor. By improving the optical train design and using real-time data analysis, improvement in axial resolution by approximately two orders of magnitudes over the focal depth of microscope objectives can be achieved. This solution has been tested for prolonged live cell imaging for fast ramping up in environmental chamber temperature and large daily swing in room temperature. In addition, this system can be used to spatial-temporally measure the surface for three-dimensional cell culture and tissue engineering, with flexibility that exceeds commercially available systems. (C) 2013 AIP Publishing LLC. [http://dx.doi.org/10.1063/1.4829616]
\end{abstract}

\section{INTRODUCTION}

Recently, one of the frontiers of biomedical research is to investigate the dynamic processes of living cells such as cell growth, motion, and differentiation, which benefits from the advances in discovering and engineering of fluorescent proteins as well as the advances in fluorescence microscopy. It contributes to many recent progresses in stem cell, tissue engineering, and cancer research. ${ }^{1}$ Microscopic studies of this type are often used to sequentially image bright field and multiple fluorescent channels at a number of optical fields repetitively for durations up to days. ${ }^{2}$ These live cell microscopes usually include motorized lateral plane stage, axial objective control, filter wheels, etc., which behave predictably. However, the focus drift is unavoidable and unpredictable. This mechanical and thermal fluctuations may come from various possible sources, including non-flat surface of specimen, lateral tilted placement of stage, environmental thermal instability of microscope body, inhomogeneous heating of optical components, cell culture vessel flex, the perfusion chamber/microfluidics chip experiments with temperature fluctuation in supplied media flow, etc. ${ }^{3}$ Such inevitable fluctuations cause the objective to lose the selected focal plane over time. Therefore, automatic focal plane detection system, also called autofocus system, has been on many biomedical scientists' wish list for many years.

Originally, the so-called autofocus systems are all software/image based software focusing algorithms that employ some type of "Figure of Merit" type metric., ${ }^{4,5}$ This quantified parameter generally describes the higher frequencies included in focused image than that out of focus. However, this conventional autofocusing mechanism is limited by response time and inability to determine focus in microscopy which

a)Electronic mail: huang.w@sustc.edu.cn construct clear images in a wide axial range, such as confocal and differential interference contrast microscopy. ${ }^{6}$ The needs to acquire multiple images could also lead to photobleaching and phototoxicity. This issue would be more severe in areas where the axial resolution is critical.

The hardware based focus drift correction systems developed by microscope manufactures utilize the red-infrared laser or light-emitting diode (LED) and complex optical detection systems to detect the axial location of a reference plane, which is usually the interface between glass and liquid where the cells residue or the air-glass interface at the bottom of the cell culture vessels. ${ }^{7,8}$ Predefined by the user is an offset between the reference plane and the axial location of desired focused image for each of the optical fields. The focus drift is mainly contributed by the relative distance between objective and reference plane, while the offset related to the fluctuation of thermal expansion of the glass thickness can be ignored. In the experiments, these focus drift correction systems repetitively find the reference plane and maintain the constant objective-to-reference plane distance through a motorized axial objective driver. Most commercial or experimentally deployed systems report repeatability of 33\%-50\% of the depth of field (DOF), which may not be enough for stringent application such as super resolution, single molecule imaging, and deconvolution optical sectioning.

Due to a number of shortcomings of commercial systems, a need for customized focus drift correction systems remains. For instance, for applications involving single molecular imaging and super resolution microscopy, it is important to maintain high axial resolution beyond the focal depth of the objective. ${ }^{9}$ Recently, alternative combinatory solutions for these super resolution imaging were proposed to precisely analyze the stack images or quadrant photodiode signals to determine the focal plane with help of submicrometer fluorescent beads conjugated to the reference interface. ${ }^{10,11}$ While 
these methods produce definitive results and high axial sensitivity, they have a number of drawbacks. First of all, the fluorescent beads conjugation is cumbersome and not suitable for most experiments, and the imaging process is also complicated and slow. Second, the focus-aid laser or LED might still interfere with highly light-sensitive species or optogenetic applications. ${ }^{12-14}$ Third, the focus drift can be used to measure three-dimensional (3D) surface that is increasingly used in cell culture and tissue engineering, ${ }^{15-17}$ which the commercial system cannot be adapted to. Finally, the commercial optical systems are complicated, difficult to manipulate, and expensive. It prohibits the adaptation by many research groups, and for converting earlier models of microscopes.

In this paper, we report a simple reflected intensity integration (RII) auxiliary autofocus system that could achieve axial resolution as high as $1 / 75$ of the DOF of objectives with commonly used objectives for live cell imaging. The red laser used to detect focal plane can be pointed away from photosensitive sample. It is a valid low cost alternative that also provides user adjustability and directly provides the data for "offthe-label" utilization of such system, such as spatial-temporal measurement of 3D surface. It can be used to track various material interfaces (glass/plastic-polydimethylsiloxane (PDMS)-liquid, etc.) and use virtually any objectives, so that it can be used in regular cell culture vessels, but also in microfluidics chips and potential 3D cell culture and tissue engineering vessels.

\section{THE OPTICAL DESIGN AND SETUP}

In our RII system, the focus position along objective optical axis is determined by the intensity of the focus-aid laser spot reflected from one of the interfaces, typically the glass/plastic to liquid interface. It is a simple design but detailed implementation, including optical setup and data analysis results in significantly higher axial resolution than commercial systems and is easy to use. The optical setup is robust and flexible enough for the average user to achieve improved performance for specific experiments.

\section{A. Optical setup and operation mode}

The autofocus system is an add-on component for an infinity-corrected microscope. The system consists of dichroic beam splitters on a kinematic mount, a focus-aid laser, a motorized module manipulating the objective relative position, and an image sensor for analyzing the projected spot. As illustrated in Fig. 1, a near infrared fiber-coupled collimated laser (CNI, MRL III 671) was guided to an infinitycorrected optical system as the focus-aid beam. The power output was attenuated by analog modulation through a linear regulated DC power supply (Array Electronic, Array3631A) according to the intensity reflected from the reference plane; light intensity of several $\mu \mathrm{W}$ is sufficient. Consequently, the collimated laser beam was guided by the standard 50/50 (reflection/transmission) ratio dichroic beam splitter I (Chroma, 50/50 Beam Splitter) into the microscope optical train (Nikon TE2000S), and then focused onto the sample by

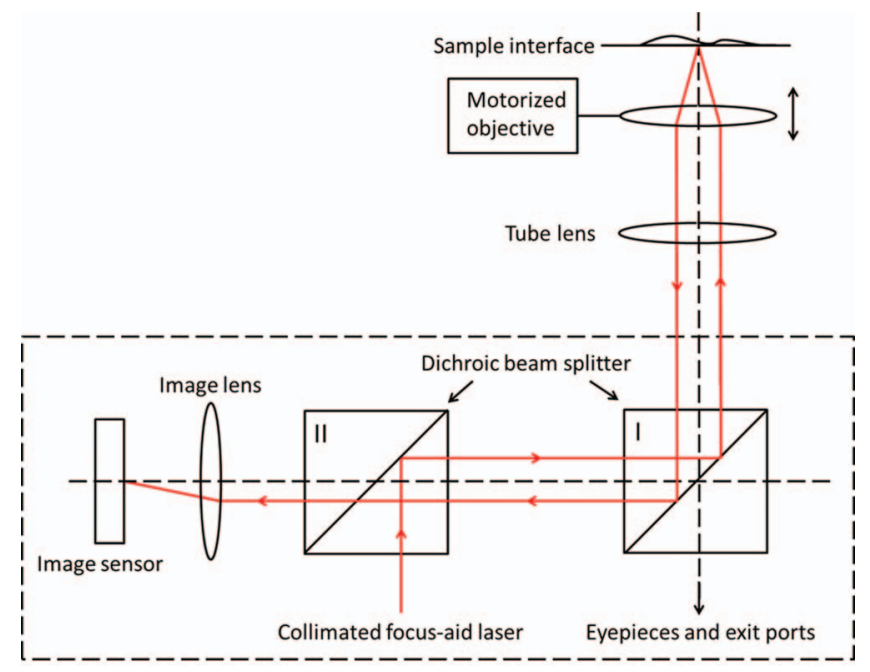

FIG. 1. A schema of reflected intensity integration auxiliary autofocus system (dashed box). Motorized objective control, image acquisition, and realtime image process are performed using LabVIEW software.

objectives (we used Nikon Plan Apo 10× NA0.45, Plan Apo $20 \times$ NA0.75, and Plan Fluor ELWD $40 \times$ NA0.6 in this study). The reflected light was then collected by the same objective lens, through beam splitter I and II, image sensor lens, and finally casted onto the CMOS image sensor (Thorlabs, DCC1545M).

By scanning along objective optical axis, the reflected Gaussian beam spot image was stepwise accumulated by the CMOS sensor, for instance, real-time parabolic fitting. The intensity from spot image reaches a peak whenever the beam focused on the sample interface. Typical images of focused spot are shown in Figure 2. With the normal incidence (Fig. 2(a)), there are many unavoidable optical surfaces within the optical train in any microscope that could contribute to the ghost images of the laser beam onto the CMOS image sensor. Such
Normal Incidence in Sensor

(a)

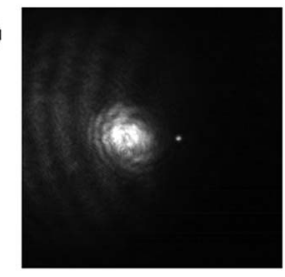

Oblique Incidence in Sensor

(c)

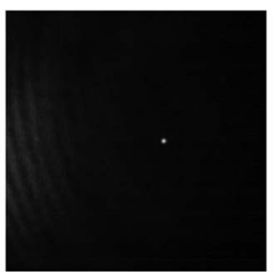

Normal Incidence in Camera

(b)

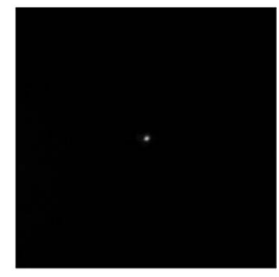

Oblique Incidence in Camera

(d)

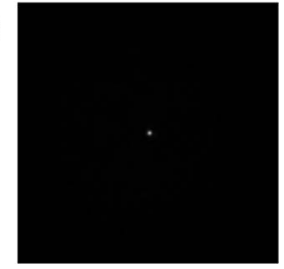

FIG. 2. Typical focused spot image. (a) and (b) show ghost images in image sensor and side port camera with normal incidence of laser beam. (c) and (d) show oblique incidence could avoid the ghost images and biology sample. All images show $200 \times 200$ pixels around the laser spot. 
interference increases the intricacy of automatically identifying the intensity of true spot image. The existing camera (SBIG, ST-402ME) on the microscope side port usually employs 1-2 internal beam splitter inside the microscope, therefore the reflections of laser beam inevitably lead to slight ghost images (Fig. 2(b)). Although using existing camera would reduce ghost image, it might not be convenient for autofocus application due to the various types of camera and communication, and it cannot be entirely independent from microscope control. Fortunately, the oblique incidence with a small angle of laser beam can easily avoid the ghost image issue, while maintaining the paraxial approximation of a Gaussian beam. We could separate the true laser spot images from the ghost images through adjusting the collimator spatial position and selecting a small area of interest (AOI) for detection (Fig. 2(c)). The oblique incidence also provides a sharper slope to improve the focusing sensitivity. Another critical advantage of this approach is to avoid the focus-aid beam from directly illuminating living cells, which is critical for minimizing phototoxicity to fragile biomedical samples or avoiding interference with other optical procedures such as optogenetics.

\section{B. Theoretical calculation}

The $\mathrm{TEM}_{00}$ fundamental mode laser beam has a Gaussian intensity profile, nevertheless real laser beam deviates from the ideal Gaussian profile which noted as "M-square" factor. The beam spherical wavefront is non-uniform distribution and changing curvature during the propagation. Therefore, the autofocus system utilizing laser as auxiliary light source could not be applied with classic geometric optics. Despite there is no analytic mathematical expression for beam output through optical fiber, it is reasonable and applicable to treat the beam intensity approximate as Gaussian distribution in both near and far field. ${ }^{18,19}$ In infinity corrected microscope optical system, the objective parfocal length is negligible compared with laser auxiliary optical train. Hence, the light propagation could be simplified by parallel laser beam incidence through a single lens.

A Gaussian beam propagating in free space follows a hyperbolic form. The minimum radius along the beam axis is noted as beam waist which is induced to describe the characteristics through an optical train. An ideal lens keeps transverse field of fundamental mode beam while changes beam waist and radius of curvature. For a thin lens, which how we treated objective, the transmitted radius of curvature of wavefront satisfies geometric optics. ${ }^{20}$ Therefore, by using complex beam parameter we could obtain the transmission formula

$$
\frac{w_{0}^{\prime 2}}{w_{0}^{2}}=\left(1-\frac{z}{f}\right)^{2}+\left(\frac{\pi w_{0}^{2}}{\lambda f}\right)^{2},
$$

where $w_{0}$ refers to incidence beam waist and $z$ is its location from lens, $w_{0}^{\prime}$ is transmitted beam waist, $\lambda$ represents wavelength, and $f$ is lens focal length. The optical train starts from fiber output, then the collimator which is a single lens with focal length of $10 \mathrm{~mm}$. The collimated laser beam with approximately Gaussian intensity distribution passing through
TABLE I. Comparison of collimated and free space laser beam.

\begin{tabular}{lrcclcc}
\hline \hline Objectives & $\begin{array}{c}\text { Focal } \\
\text { length } \\
(\mathrm{mm})\end{array}$ & $\begin{array}{c}\text { Free space } \\
\text { laser } \\
w_{0}(\mu \mathrm{m})^{\mathrm{a}}\end{array}$ & $\begin{array}{c}\text { Collimated } \\
\text { laser } \\
Z_{R}(\mu \mathrm{m})^{\mathrm{b}}\end{array}$ & $w_{0}(\mu \mathrm{m})$ & $Z_{R}(\mu \mathrm{m})$ \\
\hline $10 \times, \mathrm{NA} 0.45$ & 20 & 6.8 & 219.5 & 3 & 42.0 \\
$20 \times, \mathrm{NA} 0.75$ & 10 & 3.4 & 54.7 & 1.5 & 10.5 \\
$40 \times, \mathrm{NA} 0.60$ & 5 & 1.7 & 13.7 & 0.75 & 2.6 \\
\hline \hline
\end{tabular}

${ }^{\mathrm{a}} w_{0}$ is transmitted beam waist.

${ }^{\mathrm{b}} Z_{R}$ is Rayleigh length.

the objective and then focusing on the substrate reference interface, forms a tiny spot. The fiber-coupled laser with collimator enlarged beam waist for incidence laser into microscope optical train from $0.6 \mathrm{~mm}$ to $1.4 \mathrm{~mm}$, thus increasing the Rayleigh length $Z_{R}=\pi w_{0}^{2} / \lambda$ from $1.7 \mathrm{~m}$ to $9.5 \mathrm{~m}$ so as to improve the sensitivity. After passing through objective lens, the collimated laser beam leads to a smaller projection spot and shorter DOF defined as Rayleigh length, which makes axial intensity profile sharper compared to free space laser beam transmission. Within the range of objective DOF, long Rayleigh length means smaller axial variations, and lower sensitivities of focal plane detection. The comparison of transmitted beam waist and Rayleigh length between free space laser and collimated laser under different objectives is shown in Table I. There is an approximately quadratic relationship between the focal depth of laser (Rayleigh length) and the focal length of objective.

\section{Robust and simplified real-time data analysis}

When the reference plane lies in the perfect working distance from the objective lens, the laser spot would be in minimum size with perfect Gaussian distribution and a peak in intensity profile. For a well collimated laser beam, the minimum spot sizes on the sample and on the CMOS sensor are determined by the focal lengths of the objective and collimator lenses, as well as the numerical aperture (NA) of the optical fiber. The shape of reflected laser spot varies enormously near the focal plane, resulting in a significant change of intensity. Various peak location algorithms are robust to consistently identify the intensity peak. We chose a simple algorithm to speed up the calculation while maintaining its robustness. Specifically, we only utilize the axial intensity profile data that fall within $75 \%$ of the maximum intensity peak to fit a parabolic function to find the axial location with smallest spot size and highest intensity, which we define as the focal plane of the reflective interface. The intensity of the spot is usually computed as the sum of the intensities of an area around the spots with the pixel number determined by the optical system and detector size (Fig. 2). The performance of this system is demonstrated using a Nikon Plan Apo 20× NA0.75 objective to locate the glass-air interface of a glass slide. Twenty repetitive axial scanning are performed to estimate the resolution (Fig. 3). We use a simple curve fitting to calculate the exact axial location of laser focus for each, and the standard deviation is about $0.036 \mu \mathrm{m}$, including the errors contributed by the objective step motor, laser power fluctuation 


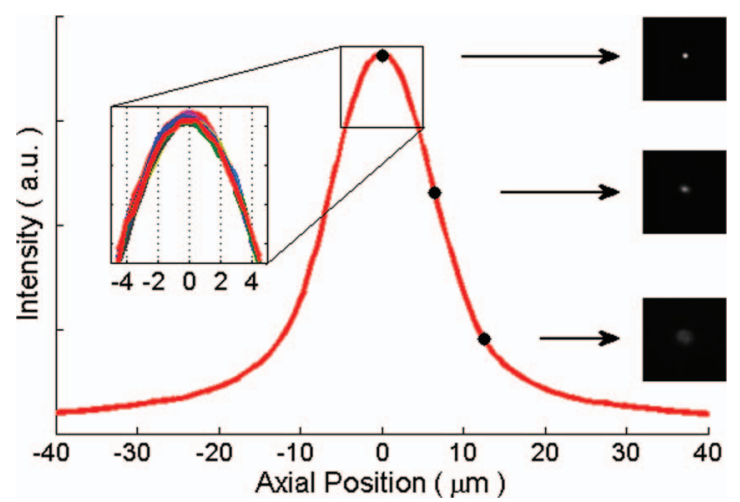

FIG. 3. Intensity profiles of axial scanning are highly repetitive. The main panel shows the curves of 20 independent axial scanning around a glass-air interface. The intensity is computed by summation of the 25 pixels covering the laser spot. To the right of the panel are actual spot images representing three different axial locations. The zoom-in plot shows the tightly correlated curves near the peak. The objective used here is Plan Apo $20 \times$ NA0.75. The axial step is $0.5 \mu \mathrm{m}$.

(Root-mean-square deviation (RMSD), 5\% for this particular model), sample vibration, etc. This repeatability is less than $2 \%$ of corresponding DOF of this objective. We repeated this test using other common objectives used for live cell imaging and the repeatability range from $1.3 \%$ to $3.8 \%$ of the DOFs (Table II). Another procedure to speed up the real-time process time and alleviate the interference of ghost images is to use a small AOI to search for brightest pixels. If the area is too small, any deviation from normal incidence angle would lead to loss of spot detection, which could also come with imperfectly leveled cell culture vessel.

\section{EXPERIMENTAL RESULTS}

\section{A. Robust tracking focus against various environmental fluctuations in live cell imaging experiment}

The most demanding area for an autofocus system is long term live cell imaging. The sources of axial focus drift mainly comes from temperature changes: (a) during the initial ramping up of the temperature in the microscope environment chamber; (b) the environmental temperature fluctuations; (c) temporal temperature perturbation such as opening the environmental chamber doors for manipulation and perfusion of biomedical media with different temperature; and (d) experimental procedures that generate large temporal temperature swings. ${ }^{21}$ Additionally, the changes of cell culture surface flex over time when plastic vessels are used will also contribute to focus drift. To test our RII system against such fluctuations, we set up a test experiment us-

TABLE II. The experimentally determined axial resolution.

\begin{tabular}{lccc}
\hline \hline Objectives & DOF $(\mu \mathrm{m})$ & Resolution $(\mu \mathrm{m})$ & Percentage $^{\mathrm{a}}(\%)$ \\
\hline 10×, NA0.45 & 5.78 & 0.22 & 3.8 \\
$20 \times$, NA0.75 & 1.93 & 0.036 & 1.9 \\
$40 \times$, NA0.60 & 2.33 & 0.031 & 1.3 \\
\hline \hline
\end{tabular}

${ }^{\text {a Percentage indicates the ratio of resolution and DOF of objective. }}$

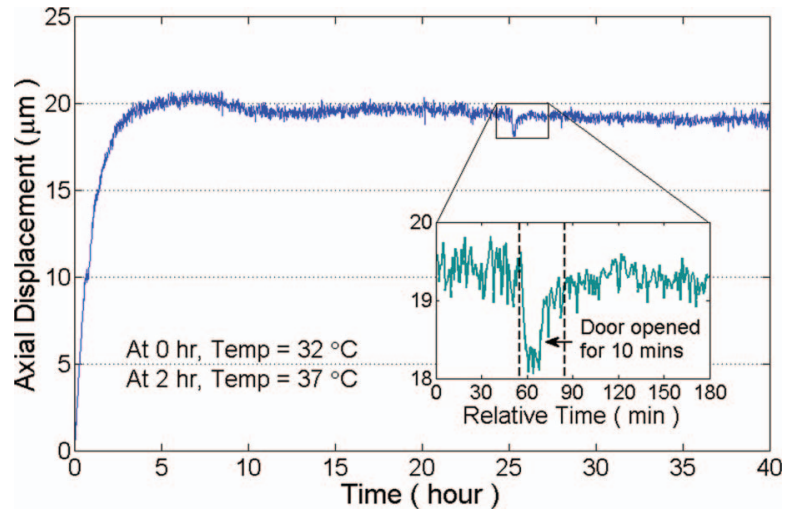

FIG. 4. Time-lapse focus drift curve corrected by the RII autofocus system. Y-axis indicates the axial displacement required to maintain focus. The time zero represents the start of focus drift correction at temperature of $32^{\circ} \mathrm{C}$ in the environment chamber near the sample. The temperature reaches a steady state of $37.0( \pm 0.1)^{\circ} \mathrm{C}$ at $2 \mathrm{~h}$. The sudden axial position drop at round 24 $\mathrm{h}$ was due to the intentionally opening the chamber door for $10 \mathrm{~min}$. The zoom-in panel indicates the drift and consequent recovery after door closing and temperature stabilizing again. The objective used here is Plan Apo $10 \times$ NA0.45.

ing a petri dish containing distilled water, fastened to the microscope stage in an environmental chamber which seals most of the microscope. An air-circling system with electrothermal heater under control of a proportional-integralderivative (PID) temperature controller (Truelab, ZNHWIV) was used to maintain the temperature inside the chamber (close to the petri dish) to around $37^{\circ} \mathrm{C}$ throughout the experiments. The objective used was Nikon Plan Apo $10 \times$ NA0.45. The initial axial position of the plastic-water interface was set at zero when the temperature reached $32^{\circ} \mathrm{C}$. The focus drift correction system was running every minute to update axial location of the interface. The experiment lasts for $40 \mathrm{~h}$ and the axial locations over time are plotted in Figure 4. Although the temperature stabilized at up to $37.0^{\circ} \mathrm{C}$ in $2 \mathrm{~h}$ with fluctuation within $0.1^{\circ} \mathrm{C}$, the focus drift exists consecutively due to the different heat expansion of the objective and other firm parts such as the stage and inhomogeneous heating in the environment inside the chamber. It increased another $5 \mu \mathrm{m}$ or so before being stabilized at around $10 \mathrm{~h}$. Our data suggest that live cell imaging should be started at least $8 \mathrm{~h}$ after the apparent temperature in the environmental chamber stabilized to minimize the drift. We also opened up the door to the chamber for $10 \mathrm{~min}$ at around $24 \mathrm{~h}$ to check if it affects temporal temperature fluctuations, and found that this particular setup need 20 additional minutes to recover after closing the door (Fig. 4, zoom-in panel). The high frequent axial location fluctuations are partially caused by the $0.22 \mu \mathrm{m}$ uncertainty with this objective and the uncertainty of the mechanical axial drive mechanisms.

\section{B. Identifying challenging interfaces in microfluidics and traction force microscopy settings}

For this class of focus tracking method, another difficult scenario is to detect the interface with minimal differences in refractive indices. Refractive indices of typical materials used in microscopy are in range 1.33 (water)-1.58 (polystyrene). 


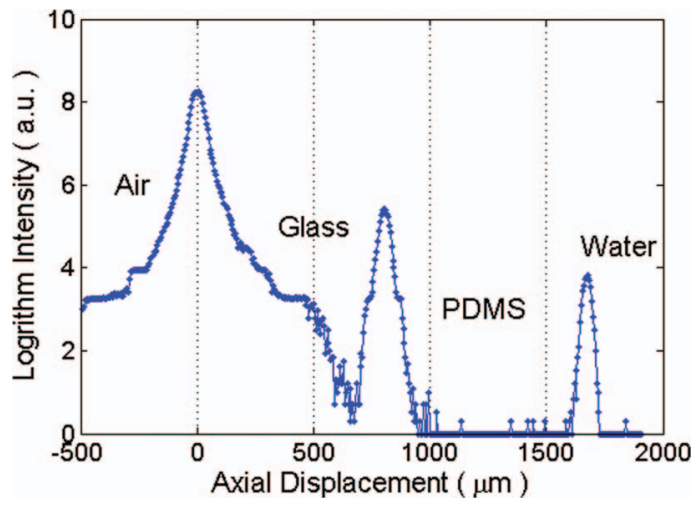

FIG. 5. The RII autofocus system detects the material interfaces with small refractive indices. From left to right, the refractive index of each medium is 1.00 for air, 1.51 for soda-lime glass, 1.42 for PDMS, and 1.33 for water. Plan Apo $10 \times$ NA0.45 is used in this experiment.

The typical air-glass/plastic interface is easy to be detected with direct reflection. We have also found the plastic/glasswater interface to be readily detected too, with index difference greater than 0.18 .

There are other interfaces with predicable difficulties. For instance, PDMS is an excellent biocompatible material widely used in microfluidics and traction force microscopy, ${ }^{22}$ which has a refractive index of approximately 1.42 with $671 \mathrm{~nm}$ wavelength. ${ }^{23}$ One such typical device would have the interfaces of glass-PDMS and PDMS-water with refractive index differences of 0.09. In addition, in this multilayer structure, the refractive light was absorbed or scattered.
Maintaining focus on the PDMS-water surface where cell adhered to and fluorescent beads conjugated to is critical to detect the tiny PDMS deformations caused by the cell. Together with quantitative measurement of the thickness of PDMS layer, we could quantitatively calculate the amplitude of traction force. ${ }^{24}$ The thickness could be different at different positions due to the viscosity and meniscus of this material during curing.

To test if our RII system can be used in this situation, we fabricated a prototype chip by spreading PDMS on chambered slide and then put the degassed PDMS prepolymer on hotplate at $80^{\circ} \mathrm{C}$ for $2 \mathrm{~h}$ for curing. We used the RII system to scan this device in order to obtain the critical parameters and maintain the focus over time. First of all, with the laser power output lowered to $1 \mu \mathrm{W}$, we can still clearly detect the three interfaces simultaneously indicated by three separate peaks (Fig. 5). The interface between PDMS and water has minimal refractive index difference, and the furthest into the sample, so the peak is the weakest. Nevertheless, it can easily be detected due to the axial sensitivity peak and excellent signal-to-noise ratio of the data.

\section{Measuring spatial-temporal modulation of 3D cell culture surface}

In recent years, cell culture is routinely performed in 3D, such as 3D surface or 3D cell clusters in stem cell differentiation, tissue engineering, and other applications. ${ }^{15-17}$ Other than relying on confocal scanning, the existing measurement (a)

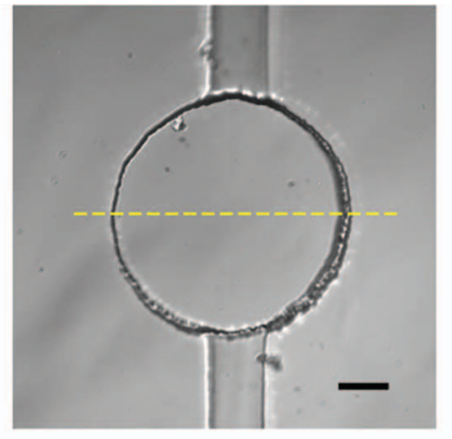

(b)

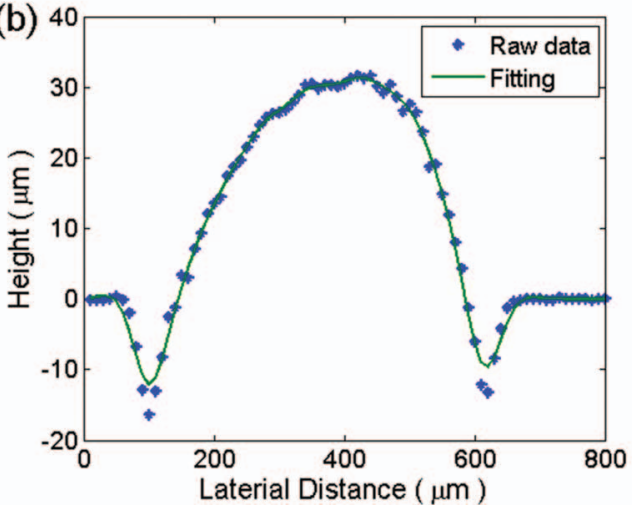

(c)

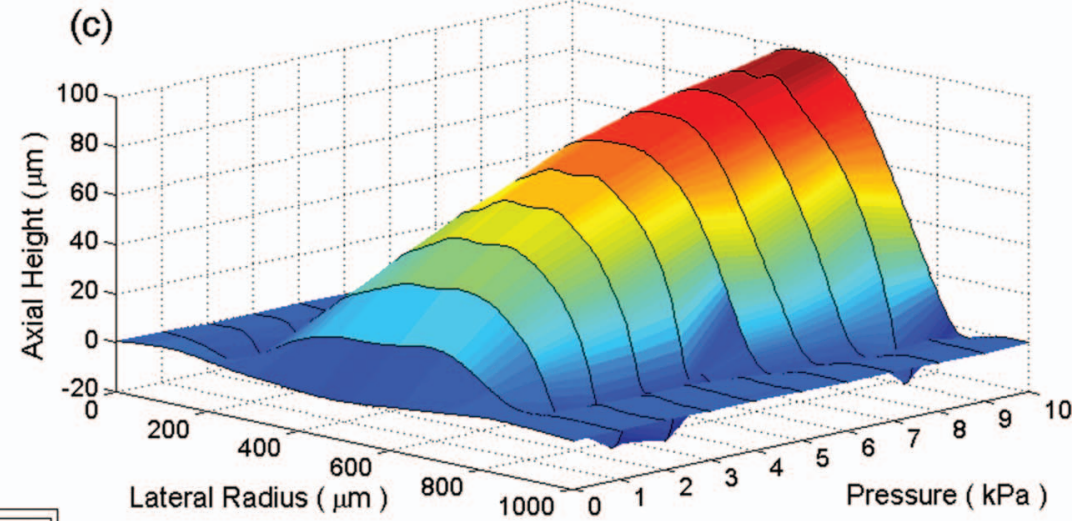

(d)

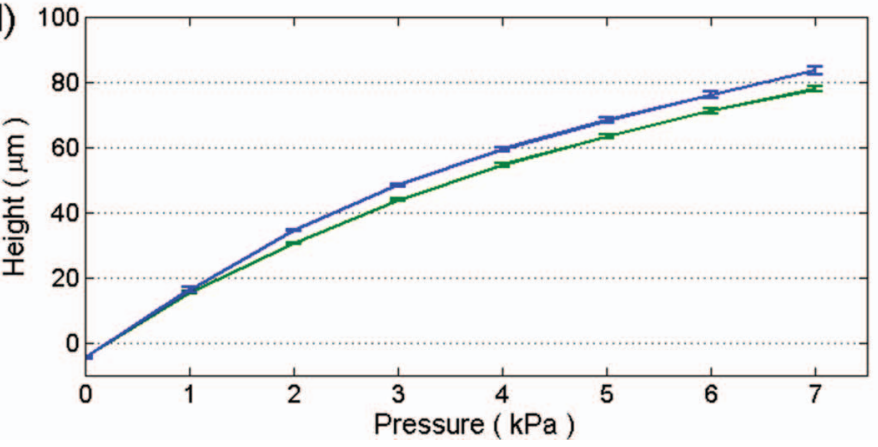

FIG. 6. Spatial-temporal profile of a pressure-tunable microlens surface measured using the RIII system. (a) Bright field image of a microlens with the path of radial scanning shown in yellow dashed line. Scale bar is $100 \mu \mathrm{m}$. (b) A typical radial-height profile under a pressure of $2 \mathrm{kPa}$. (c) A surface plot of the radial-height profiles with stepwise increased pressure. (d) The response curve of maximum height to pressure. Two curves represent two separate microlenses, and the error bars indicate standard error from three repeat measurements for each microlens. Plan Apo $10 \times$ NA0.45 is used in this experiment. 
methods for 3D geometry are not simple enough. The RII system described above should provide a valuable alternative given it provides the detailed axial scanning at different $\mathrm{x}-\mathrm{y}$ locations. In this session, we provide such a proofof-principle study using a hydraulic tunable microlens array used to generate mechanic strains to surface adherent cell. ${ }^{16}$ This device is made of PDMS with a thin layer of PDMS membrane sealing an array of micro cylinders, and flow channels connecting the cylinders (Fig. 6(a)). When a positive hydraulic pressure is applied to the flow channel, the thin membrane $(30 \mu \mathrm{m})$ on each cylinder was under equal pressure and deformed up, therefore the dome shape can be quantitatively adjusted by the pressure. In order to obtain the cross-section profile of the microlens, the previous study replicated the microlens into PDMS mold utilizing a double-molding method, which could only measure one pressure at a time, and not the same microlens and same time with cell cultured on it. We took the same microlens and performed the deformation setup through the fine tuning of the height of the water columns in the PVC tubing. At each pressure, after the pressure was stabilized, we let the focus spot run cross the entire microlens, and scan for the axial focus location at each radial location (Fig. 6(b)). The slightly asymmetry profile might be due to the imperfect alignment of the vertical channel and the round microlens. Fig. 6(c) shows a surface plot of the radial-height profiles with stepwise increased pressure. Our method directly quantified the gradual deformation of the cross-section profiles under increasing pressure and eventually reaches plateau (Fig. 6(d)).

\section{CONCLUSION AND DISCUSSION}

Previous image based or hardware based autofocus approaches require time-consuming algorithms or complicated optical controlled system, respectively. The theoretical analysis of Gaussian beam in this particular optical train casts light on a more effective and novel autofocus method. An optical fiber with matching collimator lens is chosen to collimate incidence laser and limit the focusing beam spot radius, thus significantly improves the spatial sensitivity and places no restrictions on objective selections.

The RII autofocus system has been demonstrated with high axial resolution with long term stability using various objectives. The resolution reaches $36 \mathrm{~nm}$ with a $20 \times$ objective. This performance is not only good for live cell imaging, but also sufficient for deconvolution imaging. Due to the nonlinear relation between magnification and Rayleigh length of the focused laser beam (Eq. (1)), we expect higher magnification objectives will yield significantly higher axial resolution suitable for super resolution microscopy. In addition, this system is capable to detect non-flat surface with small refrac- tive index differences, so it could also be used to measure the contour dynamics of 3D surface such as PDMS chip or 3D cell clusters, an active research area which such quantitative measurement is lacking. The low-power laser is easy to adjust either normal of oblique incidence to avoid live specimen so that it would not induce phototoxicity or interfere with optogenetic interrogation. This simple configuration is easy to retrofit into any infinite focus microscope. It also provides low cost alternative to the advanced commercial system. In addition, we believe that our customized autofocus systems not only provide better performance in many areas but also enable additional applications.

\section{ACKNOWLEDGMENTS}

We thank Jianjiang Hu and Xiongfei Fu for assistance in experiments, Hongkai Wu and Yihua Zhao for providing the microlens array. This work is supported by a Research Grants Council of Hong Kong General Research Fund (No. 767711), a National Natural Science Foundation of China (NNSFC) Fund (No. 31270887) and University of Hong Kong seed funds for basic research (Nos. 104000978 and 104001876).

${ }^{1}$ D. J. Stephens and V. J. Allan, Science 300, 82 (2003).

${ }^{2}$ M. M. Frigault, J. Lacoste, J. L. Swift, and C. M. Brown, J. Cell. Sci. 122, 753 (2009).

${ }^{3}$ F. Shen, L. Hodgson, and K. Hahn, in Methods in Enzymology, edited by I. James (Academic Press, 2006), Vol. 414, p. 620.

${ }^{4}$ F. C. Groen, I. T. Young, and G. Ligthart, Cytometry 6, 81 (1985).

${ }^{5}$ L. Firestone, K. Cook, K. Culp, N. Talsania, and K. Preston, Cytometry 12, 195 (1991).

${ }^{6}$ M. Bravo-Zanoguera, B. Von Massenbach, A. L. Kellner, and J. H. Price, Rev. Sci. Instrum. 69, 3966 (1998).

${ }^{7}$ Y. Liron, Y. Paran, N. G. Zatorsky, B. Geiger, and Z. Kam, J. Microsc. 221, 145 (2006).

${ }^{8}$ J. Peters, Nat. Methods Application Notes (2008).

${ }^{9}$ B. Huang, M. Bates, and X. W. Zhuang, Annu. Rev. Biochem. 78, 993 (2009).

${ }^{10}$ M. P. Elenko, J. W. Szostak, and A. M. van Oijen, Rev. Sci. Instrum. 81, 083705 (2010).

${ }^{11}$ A. Pertsinidis, Y. X. Zhang, and S. Chu, Nature (London) 466, 647 (2010).

${ }^{12}$ R. A. Hoebe, C. H. Van Oven, T. W. J. Gadella, P. B. Dhonukshe, C. J. F. Van Noorden, and E. M. M. Manders, Nat. Biotechnol. 25, 249 (2007).

${ }^{13}$ K. Deisseroth, Nat. Methods 8, 26 (2011).

${ }^{14}$ Y. F. Liu, Y. Zhao, X. H. Lv, Y. D. Li, X. H. Zhang, J. Zhang, L. P. Wang, and S. Q. Zen, Rev. Sci. Instrum. 83, 025116 (2012).

${ }^{15}$ K. M. Yamada and E. Cukierman, Cell 130, 601 (2007).

${ }^{16}$ Y. Zhao, J. Zhou, W. Dai, Y. Zheng, and H. Wu, J. Micromech. Microeng. 21, 054017 (2011).

${ }^{17}$ S. Y. Jung and S. J. Lee, Rev. Sci. Instrum. 83, 046102 (2012).

${ }^{18}$ D. Marcuse, J. Opt. Soc. Am. 68, 103 (1978).

${ }^{19}$ M. Young, Appl. Opt. 37, 5605 (1998).

${ }^{20}$ S. A. Self, Appl. Opt. 22, 658 (1983).

${ }^{21}$ G. Velve Casquillas et al., Lab Chip 11, 484 (2011).

${ }^{22}$ E. Gutierrez and A. Groisman, PLoS ONE 6, e25534 (2011).

${ }^{23}$ D. A. Chang-Yen, R. K. Eich, and B. K. Gale, J. Lightwave Technol. 23, 2088 (2005).

${ }^{24}$ V. Maruthamuthu, B. Sabass, U. S. Schwarz, and M. L. Gardel, Proc. Natl. Acad. Sci. U.S.A. 108, 4708 (2011). 